\title{
Error estimation for the MAP experiment
}

\author{
S. Prunet ${ }^{1}$, R. Teyssier ${ }^{2}$, S. T. Scully ${ }^{3}$, F. R. Bouchet ${ }^{4}$, and R. Gispert ${ }^{5}$ \\ 1 Canadian Institute for Theoretical Astrophysics, McLennan Labs, 60 St George Street, \\ Toronto ON M5S 3H8, Canada \\ 2 CEA/DSM/DAPNIA Service d'Astrophysique, Centre d'Études de Saclay, Bât. 709, \\ 91191 Gif-sur-Yvette Cedex, France \\ 3 Department of Physics and Astronomy, Valparaiso University, Valparaiso, IN 46383, USA \\ 4 Institut d'Astrophysique de Paris, CNRS, 94 Bld. Arago, 75014 Paris, France \\ 5 Institut d'Astrophysique Spatiale, CNRS-Université Paris-Sud, Bât. 121, 91405 Orsay, France \\ Received 2 January 2001 / Accepted 7 May 2001
}

\begin{abstract}
We report here the first full sky component separation and CMB power spectrum estimation using a Wiener filtering technique on simulated data from the upcoming MAP experiment, set to launch in early 2001. The simulations included contributions from the three dominant astrophysical components expected in the five MAP spectral bands, namely CMB radiation, Galactic dust, and synchrotron emission. We assumed a simple homogeneous and isotropic white noise model and performed our analysis up to a spherical harmonic multipole $\ell_{\max }=512$ on the fraction of the sky defined by $|b|>20^{\circ}$. We find that the reconstruction errors are reasonably well fitted by a Gaussian with an rms of $24 \mu \mathrm{K}$, but with significant deviations in the tails. Our results further support the predictions on the resulting CMB power spectrum of a previous estimate by Bouchet \& Gispert (1999), which entailed a number of assumptions this work removes.
\end{abstract}

Key words. methods: data analysis - cosmic microwave background

\section{Introduction}

The upcoming CMB satellite experiments MAP and the Planck Surveyor offer an unprecedented opportunity to measure CMB temperature fluctuations on the whole sky. A major hurdle in extracting the primary CMB signal from data, apart from noise, is the removal of the Galactic and extragalactic foregrounds. However, as the foregrounds differ from $\mathrm{CMB}$ in both frequency dependence and spatial distribution, one can reduce their residual level in a multi-frequency CMB experiment. A multifrequency, multi-resolution, Wiener filtering method to optimally extract the CMB component was developed (Bouchet et al. 1996; Tegmark \& Efstathiou 1996; Bouchet \& Gispert 1999) and lead to detailed predictions for the accuracy achievable with several experiments, and in particular, for MAP (Bouchet \& Gispert 1999). These papers were purely semi-analytical and entailed a number of simplifying approximations and assumptions. A practical implementation was performed by Bouchet et al. (1996) on the simulated data produced by Bouchet et al. (1995). These numerical studies confirmed that the residual contamination after cleaning the map is much smaller than the CMB primary signal and therefore the foregrounds may not be a major obstacle in the extraction of CMB

Send offprint requests to: S. Prunet, e-mail: prunet@cita.utoronto.ca temperature angular power spectrum if the real sky behaves similarly to the assumed model.

These simulation studies were based on filtering the data obtained on small patches of the sky of typically $10^{\circ}$ by $10^{\circ}$ angular size. They did not involve the assumption of Gaussian foregrounds since actual templates were used (e.g. portions of the Haslam and IRAS maps at respectively $408 \mathrm{GHz}$ and $100 \mu \mathrm{m})$. The results, however, could not be safely extrapolated to the full sky as a result of three facts: 1) the Wiener filter used was always optimal for the particular (small) region being analyzed instead of being an average filter for all the sky; 2) nothing could be said about modes larger than the map size; 3 ) there was no really satisfactory way of co-adding individual map results even if all maps covering the sky had been used (one problem being the use of Fourier transforms with periodic boundary conditions). Note that a similar approach based on a multi-frequency maximum entropy method in Fourier space on the same simulations leads to comparable optimistic conclusions (Hobson et al. 1998, applied to simulated MAP data by Jones et al. 1999), but with similar caveats.

In this work, we extend the Wiener filtering method to full-sky maps with a symmetric Galaxy cut $\left(|b|>20^{\circ}\right)$ and discuss our solutions to the various technical difficulties encountered. We report the results for the MAP experiment and compare them with the relevant predictions of Bouchet \& Gispert (1999). A companion paper will 
detail the implementation and give results for the Planck Surveyor experiment that requires using a much more sophisticated foreground model due to the higher resolution and higher sensitivity of that experiment.

\section{Full sky wiener filtering}

Given a set of full sky maps observed in different frequency bands (at 22, 30, 40, 60 and $90 \mathrm{GHz}$ for the MAP experiment), we want to recover the underlying CMB signal by combining the channels in an optimal way, that takes into account both the foreground contaminants and the instrumental noise.

We write the signal as the sum of the true data and the noise in each band, $\boldsymbol{d}_{\nu}=\boldsymbol{s}_{\nu}+\boldsymbol{n}_{\nu}$, where each vector component is a pixel map. The filtered signal, $\hat{\boldsymbol{s}}_{\nu}$, is a linear combination of the data, $\hat{\boldsymbol{s}}_{\nu}=W_{\nu \mu} \boldsymbol{d}_{\mu}$, where $W$ is the linear filter used in the multi-frequency reconstruction. The Wiener filter is specifically obtained by minimizing the trace of the covariance matrix of the error map defined as $\boldsymbol{e}_{\nu}=\hat{\boldsymbol{s}}_{\nu}-\boldsymbol{s}_{\nu}$. We get the usual formula $W=S(S+N)^{-1}$ where $S=\left\langle s s^{T}\right\rangle$ is the covariance matrix of the true signal and $N$ is the covariance matrix of the noise. The noise characteristics should be defined as precisely as possible for a given experiment, but the signal is a priori unknown. The prior probability given by the covariance matrix $S$ is a key ingredient in the Wiener filtering method. We assume here that the signal in a given frequency band is a linear combination of several astrophysical processes including the CMB, the different Galactic emission processes, and other extragalactic foregrounds. This can be formulated as $\boldsymbol{s}_{\nu}=A_{\nu \mathrm{p}} \boldsymbol{x}_{\mathrm{p}}$ where $\boldsymbol{x}_{\mathrm{p}}$ is a reference template for a given astrophysical process. This assumption allows us to factorize the spectral and spatial properties of the signal. The observation matrix $A$ can also account for the beam as a convolution operation on the input maps. The covariance matrix of the signal in the different frequency bands is readily obtained by $S=A C A^{T}$ where $C$ is the covariance matrix of the templates (for uncorrelated processes, it is block diagonal). The last step in the component separation is to recover from the filtered data the estimates of the astrophysical processes by a Least-Square fit to the recovered signal, namely

$\hat{\boldsymbol{x}}_{\mathrm{p}}=\left(A^{T} A\right)^{-1} A^{T} \hat{\boldsymbol{s}}_{\nu}=C A^{T}\left(A C A^{T}+N\right)^{-1} \boldsymbol{d}_{\nu}$

which is the formula for multi-frequency Wiener filtering (Bouchet et al. 1996).

One problem in analyzing full sky maps is the huge number of pixels that one must deal with. A direct pixelbased approach of the Wiener filtering technique is far beyond the capabilities of current and near-future supercomputers. One possibility is to work with coefficients of a harmonic decomposition where convolutions (of the template by the optical beam) translate into mere multiplications. Indeed previous simulation analyses all dealt with Fourier coefficients. For the full sky analysis presented here, we use instead spherical harmonics coefficients. This harmonic basis change is particularly useful if each astrophysical process can be well approximated by a homogeneous and isotropic random field with a given power spectrum. In this case, the Wiener filter can be computed for each multipole $\ell$ independently.

Here we consider that, to first order, the Galactic emission can be modelled as the sum of spatial templates for each astrophysical emission mechanism (i.e. dust, synchrotron) multiplied by their associated frequency dependence. At high galactic latitude this appears to be a good approximation (see e.g. Bouchet \& Gispert 1999, and references therein). At low galactic latitude, the situation is more complex as strong variations of the effective spectral index are observed in the Galactic disk. In addition, the presence of very bright sources at low latitude strongly violates the assumption of Gaussianity that is required for Wiener filtering to be linearly optimal, while this assumption is more reasonable at high latitude. Moreover, the presence of these bright, highly localized features is likely to ruin any component separation analysis that assumes statistical isotropy. All these points, together with the fact that CMB emission is dominant only outside the Galactic plane, indicate that a careful removal of the Galactic plane region is necessary in the analysis of full sky CMB data.

\section{Working with a galaxy cut}

\subsection{Building a new orthonormal basis on the cut sky}

The problem that arises as soon as one removes the Galactic plane region from a full sky map is the loss of orthonormality of the spherical harmonics basis (Gorski 1994). As was pointed out in the last section, a Wiener filter results from minimizing the trace of the covariance matrix $E$ of the error map. If we write for a given map $\boldsymbol{x}$ that $\boldsymbol{x}=Y \tilde{\boldsymbol{x}}$, where $Y$ are the spherical harmonics ( $Y_{l m}$ 's) and $\tilde{\boldsymbol{x}}$ are the map's multipoles, we get

$\operatorname{Tr}(E)=\left\langle\boldsymbol{e}^{\dagger} \boldsymbol{e}\right\rangle=\left\langle\tilde{\boldsymbol{e}}^{\dagger} Y^{T} Y \tilde{\boldsymbol{e}}\right\rangle \neq\left\langle\tilde{\boldsymbol{e}}^{\dagger} \tilde{\boldsymbol{e}}\right\rangle$

where $\boldsymbol{e}$ is the error map of any given process. Therefore, Wiener filtering the data in harmonic space is not equivalent anymore to Wiener filtering the data in real space. The usual way to solve this problem is to build a new orthonormal basis on the cut sky. Gorski (1994) proposed a method for the COBE-DMR data based on a Cholesky decomposition of the coupling matrix $Y^{T} Y$ of the $Y_{l m}$ 's. In the course of our work, we found that this method works well for $\ell_{\max }$ up to $40-50$, but the Cholesky decomposition failed to converge for higher multipoles because the coupling matrix becomes ill-conditioned due to numerical truncation errors. We have therefore used instead an orthonormalisation scheme based on the Singular Value Decomposition of the $Y$ matrix. The SVD can be written $Y=U D V^{T}$ where $U^{T} U=I, V^{T}=V^{-1}$, and $D$ is a diagonal matrix containing the singular values. This decomposition is numerically stable. The new basis is given by the matrix $U$ and the decomposition becomes $\tilde{\boldsymbol{x}}=U^{T} \boldsymbol{x}$ (if $\boldsymbol{x}$ can be represented on that basis, i.e. $\boldsymbol{x}=U \tilde{\boldsymbol{x}}$, then 
$\left\langle\boldsymbol{x}^{T} \boldsymbol{x}\right\rangle=\left\langle\tilde{\boldsymbol{x}}^{T} \tilde{\boldsymbol{x}}\right\rangle$ as desired). Vectors of the new basis that correspond to vanishing singular values can be dropped as their support is confined to the Galactic cut. Note that for high resolution maps, this new basis can be obtained at a reasonable computational cost (but only if the cut is symmetric). We therefore restrict ourselves to a symmetric, isolatitude Galaxy cut (with $|b|>20^{\circ}$ for example).

\subsection{Power spectrum estimator on the cut sky}

One important ingredient of the Wiener filtering technique is the covariance matrix of the astrophysical processes. One possibility is to give an analytical form of the $C(\ell)$ as a prior. In practice, however, it is more useful to compute the power spectrum directly on a first estimate of each astrophysical template (obtained for instance with an SVD method, see Bouchet \& Gispert 1999) and ultimately, do an iterative Wiener filtering by refining the estimate of the power spectra at each step. In this paper, we compute directly the power spectrum of the templates used in the simulated data, but by using only the pixels at high galactic latitudes. Tegmark (1995) described a method to obtain an optimal power spectrum estimator for each $\ell$ on an incomplete sphere by minimising the leakage between multipoles (see Tegmark 1995, for details). We use this method to design "optimal masks" or "apodising functions" to compute the $C(\ell)$ on our Galaxy cut templates. Our implementation was designed to work in the framework of the orthonormal basis on the cut sky described in the last section. An intermediate possibility would be to exploit the smoothness of the different power spectra. The computation of the power spectra in terms of bandpowers (Bond et al. 2000) might offer in this respect a good compromise between spectral resolution and computational speed, but we did not test it.

\section{Numerical techniques}

In this work, we use extensively the HEALPix pixelisation scheme $^{1}$. We worked at a resolution $n_{\text {side }}=256$ with a pixel size of $13.7 \mathrm{arcmin}^{2}$. This pixelisation choice is a little too coarse to extract all the CMB information from MAP since its $90 \mathrm{GHz}$ channel has a $12.8 \operatorname{arcmin} F W H M$ beam. As we shall see, however, it is enough to capture the essential features of the Wiener filtering possibilities for the MAP experiment.

Due to the constraint that our Galaxy cut is symmetric with respect to the Galactic plane, we can address each $m$ for odd and even $\ell$ separately. This rotational symmetry reduces the computational complexity from $O\left(\mathrm{~N}_{\mathrm{pix}}^{2}\right)$ down to $O\left(\mathrm{~N}_{\text {pix }}^{3 / 2}\right)$. Computing the new basis represents 20 min on a single processor of a SGI O2000 parallel system for $n_{\text {side }}=256$ and $\ell_{\max }=512$. Recomputing the new basis each time we need to analyze a map is not very efficient. We therefore store permanently the new coordi-

\footnotetext{
1 see http://www.eso.org/ kgorski/healpix/

2 Healpix divides the sphere in 12 main regions, each being hierarchically divided in $n_{\text {side }}^{2}$ pixels.
}

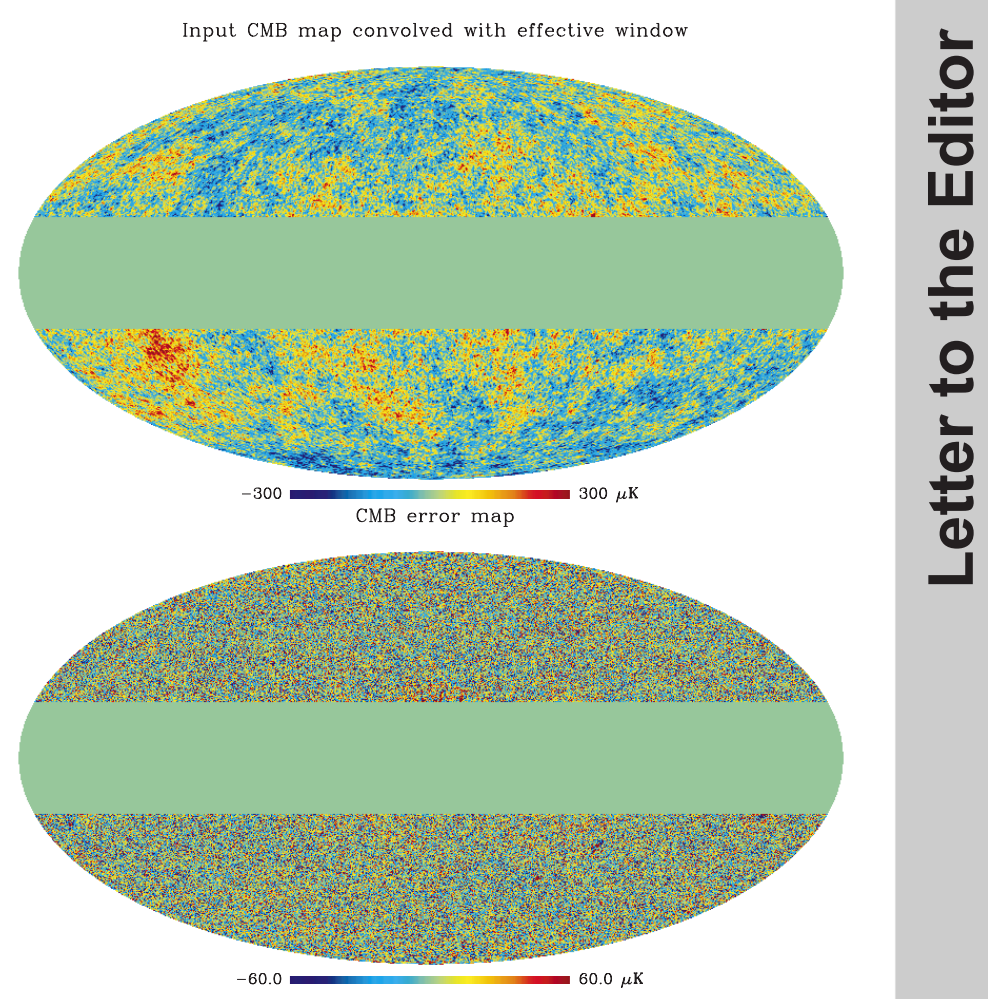

Fig. 1. Upper panel: the CMB input map, convolved with the effective window function of the experiment. Lower panel: the CMB error map. Note the different scale used here. the error is mostly white noise, except near the galactic cut. Several bright spots are also visible.

nates, which requires approximately 1 GB space in double precision. Computing the "optimal masks" for power spectrum estimates is much more computationally expensive. The core of the method is to solve a General Eigenvalue Problem $(A x=\lambda B x)$ (Tegmark 1995), and to compute only the first eigenvector. This process takes $45 \mathrm{~min}$ on the same parallel computer using 10 processors running in parallel under MPI. The storage of these "optimal masks" also requires approximately $1 \mathrm{~GB}$ in double precision. Note that storage requirements are important here, since extrapolating our results to $n_{\text {side }}=1024$ and $\ell_{\max }=2048$ leads to a total computational time of 48 hours with 10 processors and a total memory requirement of $128 \mathrm{~GB}$. We have, however, identified possible ways to decrease the CPU and memory requirements each by a factor of 4 .

\section{Results and discussion}

In this work we modelled the Galactic emission to be composed only of synchrotron and dust emission. Other forms of emission such as uncorrelated free-free emission or point and SZ sources were left out for simplification of the interpretation of the results. We will address those additional components in a future paper. The dust emission was simulated using the Finkbeiner et al. DIRBE/IRAS composite $100 \mu \mathrm{m}$ map (Finkbeiner et al. 1999), and the synchrotron emission using the Haslam $408 \mathrm{MHz}$ map (Haslam et al. 1982) with spectral index -0.9. The dust was assumed to have a single $18 \mathrm{~K}$ component with 


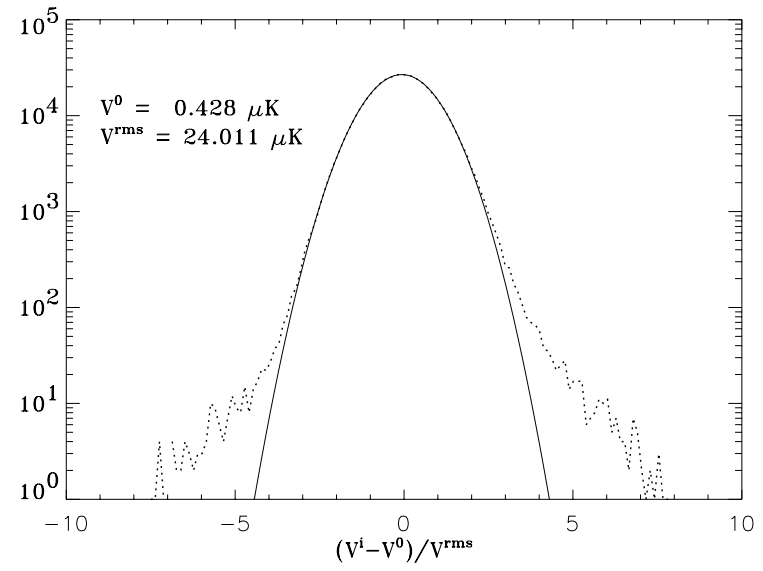

Fig. 2. Distribution of the $\mathrm{CMB}$ map reconstruction error (dotted line), obtained by subtracting the recovered CMB map from the input CMB map smoothed with the effective window function for the CMB ("quality factor", see Bouchet \& Gispert 1999) produced by Wiener filtering (see text). Also shown is the best-fit Gaussian (solid line) with its mean and rms. Note that the non-zero mean value shows the small level of contamination of the reconstructed CMB by the foregrounds monopoles. It will however not be an issue for the real observations since MAP is a differential experiment.

emissivity index of 2 , together with a correlated free-free like component of spectral index -0.15 .

Figure 1 shows the input CMB map, smoothed at the expected resolution of the reconstructed map, and the error map, which is the difference of the input CMB map and the recovered one. As the latter shows, the overall reconstruction is excellent, although a few glitches associated with low galactic latitude HII clouds are visible close to the Galaxy cut. This justifies a posteriori the removal of the Galactic plane from our analysis. Figure 2 gives the distribution of the reconstructed errors, whose bulk is well approximated by a Gaussian distribution with an rms of $24 \mu \mathrm{K}$. Note though the low level wings that are likely associated with the residual imprint of HII clouds. The corresponding pixels would probably have to be dropped out from any ensuing analysis.

An important feature of Wiener filtering is that the quality factor $(\mathrm{s})^{3}$ of the instrument is an output of the analysis. The expected resolution of the reconstructed map is thus known directly and can be accounted for in later stages of the statistical analysis. Indeed we used it here to convolve our input CMB map to the expected resolution of the recovered map.

For cosmological purposes, the most important statistic is the angular power spectrum of the CMB. We therefore show the power spectrum of the recovered CMB maps together with the spectrum of the reconstruction error in Fig. 3. The spectra are computed for each $\ell$ using the

3 The quality factors are the generalized instrumental window functions for each recovered astrophysical process, taking into account the angular resolution and the detector noise of every channel, as well as the contamination of other processes, see Bouchet \& Gispert (1999).

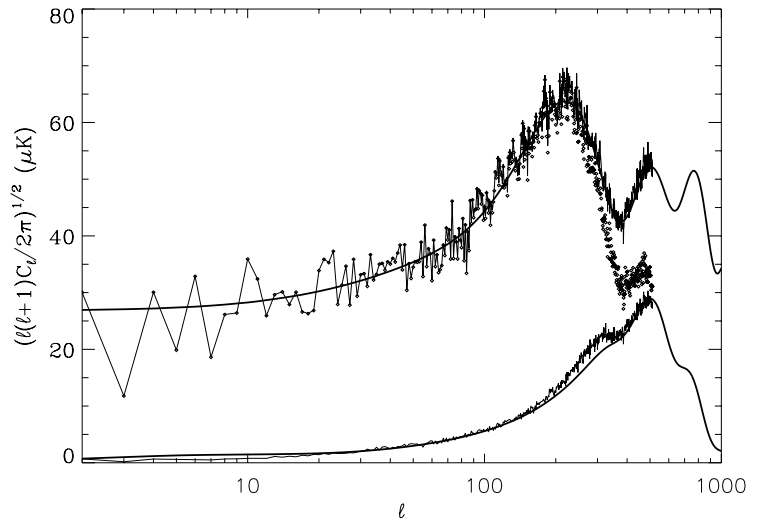

Fig. 3. Comparison of the input sCDM angular power spectrum (upper thick solid line) to that of the reconstructed (Wiener-filtered, hence smoothed) CMB map (dots, middle noisy curve). The latter can be used to obtain an unbiased estimate of the input spectrum (thin solid line, on top of the input spectrum) by using the quality factor produced by the analysis (see text). The lower (thick solid) curve shows the semi-analytical prediction of the spectrum of the reconstruction error by Bouchet \& Gispert (1999), together with the actual estimate (thin solid) from our full-sky error map.

minimal leakage apodising masks on the incomplete sphere (see Sect. 3.2). The curves abruptly end at $\ell=512$, the resolution limit of the present analysis, where incidentally the error is of the order of the CMB signal. Note however that a box car averaging of the $C(\ell)$ with $\Delta \ell$ around 10 will reduce the noise level, and therefore a strong (and thus useful) cosmological signal is still expected above $\ell=512$, likely up to $\ell=1024$. We will extend this work in a companion paper to a spatial resolution of at least $\ell=1024$. The main conclusion of the current work is that we confirm the semi-analytical predictions of Bouchet \& Gispert (1999), but using this time a full sky analysis of simulated MAP data.

Acknowledgements. Simulations and map analysis were performed on MAGIQUE, an SGI 0200014 processors parallel system at IAP. The authors would like to thank Didier Vibert for his help on this project.

\section{References}

Bond, J. R., Jaffe, A. H., \& Knox, L. 2000, ApJ, 533, 19

Bouchet, F. R., \& Gispert, R. 1999, New Astron., 4, 443

Bouchet, F. R., Gispert, R., Aghanim, N., et al. 1995, Space Sci. Rev., 74, 37

Bouchet, F. R., Gispert, R., \& Puget, J.-L. 1996, in Unveiling the Cosmic Infrared Background, ed. E. Dwek (Baltimore), AIP Conf. Proc., 348, 255

Finkbeiner, D. P., Davis, M., \& Schlegel, D. J. 1999, ApJ, 524, 867

Gorski, K. M. 1994, ApJ, 430, L85

Haslam, C. G. T., Stoffel, H., Salter, C. J., \& Wilson, W. E. 1982, A\&AS, 47, 1

Hobson, M. P., Jones, A. W., Lasenby, A. N., \& Bouchet, F. R. 1998, MNRAS, 300, 1

Jones, A. W., Hobson, M. P., \& Lasenby, A. N. 1999, MNRAS, 305,898

Tegmark, M. 1995, ApJ, 455, 429

Tegmark, M., \& Efstathiou, G. 1996, MNRAS, 281, 1297 